\title{
Controller Design With Regional \\ Pole Constraints: Hyperbolic and Horizontal Strip Regions
}

\author{
Y. William Wang ${ }^{1}$ and Dennis S. Bernstein \\ Department of Aerospace Engineering \\ The University of Michigan \\ Ann Arbor, MI 48109-2140 \\ (313) 764-3719, (313) 763-0578(FAX)
}

\begin{abstract}
In this paper we consider the design of robust controllers with closed-loop poles constrained to lie in specified regions in the left half complex plane. The paper focuses in particular on hyperbolic and horizontal strip regions. The hyperbolic region places a lower bound on the damping ratio whereas the horizontal strip region places an upper bound on the natural frequency of the closed-loop system. Each constraint region is characterized by a pair of matrix rootclustering equations. These equations, which govern the response of the closed-loop system, are utilized in conjunction with a steady state quadratic performance criterion. By applying fixed-structure synthesis techniques, we obtain feedback controllers that achieve the desired performance properties along with suboptimal closed-loop performance.
\end{abstract}

\section{Nomenclature}

\begin{tabular}{|c|c|}
\hline $\mathcal{R}, \mathcal{R}^{m \times l}$ & real numbers, $m \times l$ real matrices \\
\hline $\mathcal{C}, \mathcal{C}^{m \times l}$ & complex numbers, $m \times l$ complex matrices \\
\hline $\mathcal{E}, \operatorname{tr}$ & expectation, trace operator \\
\hline$I_{r}$ & $r \times r$ identity matrix \\
\hline$\otimes, \oplus, U_{n}$ & $\begin{array}{l}\text { Kronecker product, Kronecker sum, } n \times n \\
\text { permutation matrix; as defined in Ref. } 12\end{array}$ \\
\hline$\sigma(A), \bar{\lambda}$ & spectrum of $A$, complex conjugate of $\lambda \in \mathcal{C}$ \\
\hline$x, u, y, x_{\mathrm{c}}$ & $n, m, l$ and $n_{c^{-}}$dimensional vectors \\
\hline$w$ & $d$-dimensional standard white noise \\
\hline$A, B, C$ & $n \times n, n \times m$ and $l \times n$ dimensional matrices \\
\hline$D_{1}, D_{2}, K$ & $n \times d, l \times d$ and $m \times l$ dimensional matrices \\
\hline$A_{c}, B_{c}, C_{c}$ & $n_{c} \times n_{c}, n_{c} \times l$ and $m \times n_{c}$ matrices \\
\hline$R_{1}, R_{12}, R_{2}$ & $n \times n, n \times m$ and $m \times m$ weighting matrices \\
\hline$a, b$ & hyperbolic region parameters \\
\hline$A_{s}, V_{s}$ & $A+B K C, D_{1} D_{1}^{\mathrm{T}}>0$ \\
\hline$R_{s}$ & $R_{1}+R_{12} K C+\left(R_{12} K C\right)^{\mathrm{T}}+C^{\mathrm{T}} K^{\mathrm{T}} R_{2} K C$ \\
\hline$A_{r}, A_{e}$ & $A+B C_{c}, A+B_{c} C$ \\
\hline$V_{1}, V_{12}, V_{2}$ & $D_{1} D_{1}^{\mathrm{T}}>0, D_{1} D_{2}^{\mathrm{T}}, D_{2} D_{2}^{\mathrm{T}}$ \\
\hline$V_{e}$ & $V_{1}-B_{c} V_{12}^{\mathrm{T}}-V_{12} B_{c}+B_{c} V_{2} B_{c}^{\mathrm{T}}$ \\
\hline$R_{r}$ & $R_{1}+R_{12} C_{c}+\left(R_{12} C_{c}\right)^{\mathrm{T}}+C_{c}^{\mathrm{T}} R_{2} C_{c}$ \\
\hline$R_{d}, A_{d}$ & {$\left[\begin{array}{cc}R_{1} & R_{12} C_{c} \\
C_{c}^{\mathrm{T}} R_{12}^{\mathrm{T}} & C_{c}^{\mathrm{T}} R_{2} C_{c}\end{array}\right], \quad\left[\begin{array}{cc}A & B C_{c} \\
B_{c} C & A_{c}\end{array}\right.$} \\
\hline$V_{d}$ & {$\left[\begin{array}{cc}V_{1} & V_{12} B_{c}^{\mathrm{T}} \\
B_{c} V_{12}^{\mathrm{T}} & B_{c} V_{2} B_{c}^{\mathrm{T}}\end{array}\right]$} \\
\hline
\end{tabular}

\footnotetext{
${ }^{1}$ AIAA Member

${ }^{2}$ This research is supported in part by the Air Force Office of Scientific Research under grant F49620-92-J-0127.
}

Copyright (C) 1992 American Institute of Aeronautics and Astronautics, Inc All rights reserved.

\section{Introduction}

In Ref. 1, fixed-structure synthesis techniques were used to design feedback controllers that place the closed-loop poles within specified regions in the open left half plane. Specifically, circular, elliptic, parabolic, vertical strip and sector regions were considered with both static and dynamic output feedback controllers. The purpose of the present paper is to extend the results of Ref. 1 by considering two regions that were not considered in Ref. 1, namely, hyperbolic and horizontal strip regions. In practice, the hyperbolic region, which was considered in Refs. 2-9, imposes a lower bound on the damping ratio of the closed-loop poles, while the horizontal strip region, briefly discussed in Ref. 10, imposes an upper bound on the natural frequencies of the closed-loop poles. The complicating aspect of both of these regions is that each region is reflected into the right half plane. Hence, it is necessary to exclude from considering the right half portion of the constraint region.

Our development proceeds, in Section II, with an analysis of the hyperbolic constraint region. We then turn, in Section III, to the problem of controller synthesis with static output feedback controller, including the specialization to full state feedback. Analogous results for full-order dynamic compensation with regulator/estimator separation are given in Section IV. In Section V, we show results for fixed-order dynamic compensation with an optimal projection condition. We then, in Section VI, turn our attention briefly to the horizontal strip region. Results from a given numerical example is shown in Section VII. Finally, some conclusions and directions for further research are given in Section VIII.

\section{Characterization of the Hyperbolic Constraint Region}

In this section we characterize the hyperbolic region, shown in Figure $1, \mathcal{H}_{\mathcal{L}}(a, b)$. The two-sided hyperbolic region $\mathcal{H}(a, b)$ is defined by

$$
\mathcal{H}(a, b) \triangleq\left\{\lambda \in \mathcal{C}: \frac{(\operatorname{Re} \lambda)^{2}}{a^{2}}-\frac{(\operatorname{Im} \lambda)^{2}}{b^{2}}>1\right\},
$$

where $a$ and $b$ are positive real numbers. In order to specify the left half region which is of interest for stability, we focus on the subset

$$
\mathcal{H}_{\mathcal{L}}(a, b) \triangleq\{\lambda \in \mathcal{H}(a, b): \operatorname{Re} \lambda<0\},
$$

which corresponds to the left branch of the hyperbola. It is often convenient to write $\lambda=-\zeta \omega_{n}+j \omega_{d}$, where $0 \leq \zeta \leq 1$ and $\omega_{\mathrm{d}}=\omega_{\mathrm{n}} \sqrt{1-\zeta^{2}}$. It is also known that the settling time 
is related to $\operatorname{Re}(\lambda)$. In practice, design criteria may involve the damping ratio $\zeta$ and the reciprocal of the settling time $\eta=\zeta \omega_{\mathbf{n}}$. The constraint $\zeta \geq \zeta_{\min }$ and $\eta \geq \eta_{\min }$ can be enforced by the hyperbola parameters $a, b$ by choosing

$$
\begin{gathered}
a=\eta_{\mathrm{min}}, \\
b=\frac{\eta_{\min }}{\zeta_{\min }} \sqrt{1-\zeta_{\min }^{2}} .
\end{gathered}
$$

The following result provides an alternative characterization of $\mathcal{H}(a, b)$.

Lemma 2.1. The set $\mathcal{H}(a, b)$ is equivalent to

$$
\mathcal{H}(a, b) \triangleq\left\{\lambda \in \mathcal{C}: 1+2 \delta\left(\operatorname{Re} \lambda^{2}\right)+\gamma|\lambda|^{2}<0\right\},
$$

where

$$
\delta \triangleq-\frac{a^{2}+b^{2}}{4 a^{2} b^{2}}, \quad \gamma \triangleq \frac{a^{2}-b^{2}}{2 a^{2} b^{2}}
$$

Proof. The result follows from algebraic manipulation.

Proposition 2.1. Let $A \in \mathcal{R}^{n \times n}$, let $V_{h} \in \mathcal{R}^{n \times n}$ be positive definite and let $\delta$ and $\gamma$ be real numbers such that $\delta<0$ and $2 \delta<\gamma<-2 \delta$. Then, if there exists an $n \times n$ positive definite matrix $Q_{h}$ satisfying

$$
0=Q_{h}+\delta\left(A^{2} Q_{h}+Q_{h} A^{2 \mathrm{~T}}\right)+\gamma A Q_{h} A^{\mathrm{T}}+V_{h},
$$

then $\sigma(A) \subset \mathcal{H}(a, b)$, where

$$
a \triangleq\left(\frac{1}{-2 \delta-\gamma}\right)^{1 / 2}, \quad b \triangleq\left(\frac{1}{-2 \delta+\gamma}\right)^{1 / 2} .
$$

Proof. Let $\bar{\lambda}$ be an eigenvalue of $A^{\mathrm{T}}$ such that $A^{\mathrm{T}} x=$ $\bar{\lambda} x$. Then, forming the equation $x^{*}(2) x$ yields

$0=x^{*} Q_{h} x+\delta\left(x^{*} A^{2} Q_{h} x+x^{*} Q_{h} A^{2 \mathrm{~T}} x\right)+\gamma x^{*} A Q_{h} A^{\mathrm{T}} x+x^{*} V_{h} x$

which further implies that

$$
1+\delta \operatorname{Re} \lambda^{2}+\gamma|\lambda|^{2}=-\frac{x^{*} V_{h} x}{x^{*} Q_{h} x} .
$$

Since $V_{h}>0$ and $Q_{h}>0$, it follows that both sides of (5) are negative. Hence, $\sigma(A) \subset \mathcal{H}(a, b)$.

Note that $\mathcal{H}(a, b)$ includes regions lying in the open left half plane $\mathcal{C}^{-}$and in the open right half plane $\mathcal{C}^{+}$. Proposition 2.1 applies to all of $\mathcal{H}(a, b)$, not just $\mathcal{H}_{\mathcal{L}}(a, b)$. Considering stability, we now combine the standard Lyapunov equation with (2). Thus, the characteristic roots will be constrained to lie inside the hyperbolic constraint region.

Theorem 2.1. Let $A, V, V_{h}, Q, Q_{h} \in \mathcal{R}^{n \times n}$ and let $V$ and $V_{h}$ be positive definite matrices. Then, if there exist positive definite matrices $Q, Q_{h}$, and real numbers $\delta$ and $\gamma$ such that $\delta<0$ and $2 \delta<\gamma<-2 \delta$ satisfying

$$
\begin{gathered}
0=Q_{h}+\delta\left(A^{2} Q_{h}+Q_{h} A^{2 \mathrm{~T}}\right)+\gamma A Q_{h} A^{\mathrm{T}}+V_{h}, \\
0=A Q+Q A^{\mathrm{T}}+V,
\end{gathered}
$$

then $\sigma(A) \subset \mathcal{H}_{\mathcal{L}}(a, b)$, where $a, b$ are given by $(3)$.

Proof. It follows from (6) and Proposition 2.1 that $\sigma(A) \subset \mathcal{H}(a, b)$. In addition, (7) implies $\sigma(A) \subset \mathcal{C}^{-}$. Hence, $\sigma(A) \subset \mathcal{H} \cap \mathcal{C}^{-}=\mathcal{H}_{\mathcal{L}}(a, b)$.

Remark 2.1. Equation (6) and (7) characterize the hyperbolic constraint region, where $V$ and $V_{h}$ are positive definite but otherwise arbitrary.
Lemma 2.2. Let $A$ be stable and $\sigma(A)=\left\{\lambda_{1}, \ldots, \lambda_{\mathbf{n}}\right\}$. Define $G \triangleq\left[g_{\text {ik }}\right]$ by

$$
g_{\mathrm{ik}}=\frac{-1}{\lambda_{\mathrm{i}}+\bar{\lambda}_{\mathrm{k}}} .
$$

Then $G$ is a non-negative definite matrix.

Proof. Since $A$ is stable, it follows that the eigenval ues of $A$ lie in the open left half plane. Thus define the stable diagonal matrix $\Gamma \triangleq \operatorname{diag}\left(\lambda_{1}, \ldots, \lambda_{n}\right)$. Then the Lyapunov equation

$$
0=\Gamma G+G \Gamma^{*}+E,
$$

has the non-negative definite solution

$$
G=\int_{0}^{\infty} \mathrm{e}^{\Gamma \mathrm{t}} E \mathrm{e}^{\Gamma * \mathrm{t}} \mathrm{dt}=\left[\frac{-1}{\lambda_{\mathrm{i}}+\bar{\lambda}_{\mathrm{k}}}\right]_{\mathrm{i}, \mathrm{k}=\mathbf{1}, \ldots, \mathrm{n}},
$$

where $\mathrm{E}$ has all 1's in its entries and '*' denotes the Hermitian operator.

Let $\mathcal{A}, \overline{\mathcal{A}} \in \mathcal{R}^{2 n \times 2 n}$ be defined as follows:

$$
\begin{gathered}
\mathcal{A} \triangleq I+\delta\left(A^{2} \oplus A^{2}\right)+\gamma A \otimes A \\
\tilde{\mathcal{A}} \triangleq-I-\frac{1}{\delta}(A \oplus A)^{-2}[I+(\gamma-2 \delta) A \otimes A] .
\end{gathered}
$$

Proposition 2.2 Let $\delta$ and $\gamma$ be real numbers such that $\delta<0$, and $2 \delta<\gamma<-2 \delta$ and let $a, b$ be given by (3). Then the following statements hold.

(i) Suppose $a \geq b$. Then $\mathcal{A}$ and $A$ are asymptotically stable if and only if $\sigma(A) \subset \mathcal{H}_{\mathcal{L}}(a, b)$.

(ii) Suppose $a<b$. Then $\tilde{\mathcal{A}}$ and $A$ are asymptotically stable if and only if $\sigma(A) \subset \mathcal{H}_{\mathcal{L}}(a, b)$.

Proof. We will start with the proof of statement ( $i$ ). Let $\sigma(A)=\left\{\lambda_{1}, \lambda_{2}, \ldots, \lambda_{n}\right\}$ and note that each eigenvalue of $\mathcal{A}$ can be expressed as

$$
1+\delta\left(\lambda_{i}^{2}+\lambda_{k}^{2}\right)+\gamma \lambda_{i} \lambda_{k}
$$

This result is directly obtained from Theorem 7 in Ref. 2. Then letting $\lambda_{i} \triangleq x_{i}+\mathrm{j} y_{i}, \lambda_{k} \triangleq x_{k}+\mathrm{j} y_{k}$, the real part of each eigenvalue of $\mathcal{A}$ is given by

$$
\begin{gathered}
\operatorname{Re}\left\{1+\delta\left(\lambda_{i}^{2}+\lambda_{k}^{2}\right)+\gamma \lambda_{i} \lambda_{k}\right\}=\operatorname{Re}\left\{1-\frac{a^{2}+b^{2}}{4 a^{2} b^{2}}\left(\lambda_{i}^{2}+\lambda_{k}^{2}\right)\right. \\
\left.+\frac{a^{2}-b^{2}}{2 a^{2} b^{2}} \lambda_{i} \lambda_{k}\right\} \\
=1-\frac{a^{2}+b^{2}}{4 a^{2} b^{2}}\left(x_{i}^{2}+x_{k}^{2}-y_{i}^{2}-y_{k}^{2}\right)+\frac{a^{2}-b^{2}}{2 a^{2} b^{2}}\left(x_{i} x_{k}-y_{i} y_{k}\right) \\
=1-\left\{\frac{1}{4 b^{2}}\left[\left(x_{i}-x_{k}\right)^{2}-\left(y_{i}-y k\right)^{2}\right]+\frac{1}{4 a^{2}}\left[\left(x_{i}+x_{k}\right)^{2}-\left(y_{i}+y_{k}\right)^{2}\right]\right\} .
\end{gathered}
$$

To prove sufficiensy of $(i)$, we note that since $\sigma(A) \subset \mathcal{H}_{\mathcal{L}}(a, b)$ it follows that $\mathrm{A}$ is stable and that

$$
\frac{x_{i}^{2}}{a^{2}}-\frac{y_{i}^{2}}{b^{2}}>1, \quad \frac{x_{k}^{2}}{a^{2}}-\frac{y_{k}^{2}}{b^{2}}>1,
$$

where $x_{i}<-a$ and $x_{k}<-a$. This further implies that

$$
\begin{aligned}
& \frac{\left(x_{i}+x_{k}\right)^{2}}{a^{2}}-\frac{\left(y_{i}+y_{k}\right)^{2}}{b^{2}}>2+\frac{2 x_{i} x_{k}}{a_{2}}-\frac{2 y_{i} y_{k}}{b^{2}}, \\
& \frac{\left(x_{i}-x_{k}\right)^{2}}{a^{2}}-\frac{\left(y_{i}-y_{k}\right)^{2}}{b^{2}}>2-\frac{2 x_{i} x_{k}}{a_{2}}+\frac{2 y_{i} y_{k}}{b^{2}} .
\end{aligned}
$$

Now, since $a \geq b$, the following inequalities hold 


$$
\begin{aligned}
& \frac{\left(x_{i}+x_{k}\right)^{2}}{a^{2}}-\frac{\left(y_{i}+y_{k}\right)^{2}}{a^{2}}>2+\frac{2 x_{i} x_{k}}{a_{2}}-\frac{2 y_{i} y_{k}}{b^{2}}, \\
& \frac{\left(x_{i}-x_{k}\right)^{2}}{b^{2}}-\frac{\left(y_{i}-y_{k}\right)^{2}}{b^{2}}>2-\frac{2 x_{i} x_{k}}{a_{2}}+\frac{2 y_{i} y_{k}}{b^{2}} .
\end{aligned}
$$

Then, we have

$$
\begin{gathered}
\operatorname{Re}\left\{1+\delta\left(\lambda_{i}^{2}+\lambda_{k}^{2}\right)+\gamma \lambda_{i} \lambda_{k}\right\} \\
=1-\left\{\frac{1}{4 b^{2}}\left[\left(x_{i}-x_{k}\right)^{2}-\left(y_{i}-y_{k}\right)^{2}\right]+\frac{1}{4 a^{2}}\left[\left(x_{i}+x_{k}\right)^{2}-\left(y_{i}+y_{k}\right)^{2}\right]\right\} \\
<1-\frac{1}{4}\left\{4+\frac{2 x_{i} x_{k}}{a^{2}}-\frac{2 x_{i} x_{k}}{a^{2}}-\frac{2 y_{i} y_{k}}{b^{2}}+\frac{2 y_{i} y_{k}}{b^{2}}\right\}=0 .
\end{gathered}
$$

Hence $\mathcal{A}$ and $A$ are both stable. To prove necessary of $(i)$, since $\mathcal{A}$ and $A$ are stable, it follows that

$$
\begin{gathered}
\operatorname{Re}\left\{1+\delta\left(\lambda_{i}^{2}+\lambda_{k}^{2}\right)+\gamma \lambda_{i} \lambda_{k}\right\} \\
=1-\left\{\frac{1}{4 b^{2}}\left[\left(x_{i}-x_{k}\right)^{2}-\left(y_{i}-y_{k}\right)^{2}\right]+\frac{1}{4 a^{2}}\left[\left(x_{i}+x_{k}\right)^{2}-\left(y_{i}+y_{k}\right)^{2}\right]\right\} \\
<0 .
\end{gathered}
$$

If $\lambda_{i}=\bar{\lambda}_{k}$ then let $x_{i}=x_{k}$ and $y_{i}=-y_{k}$ and thus

$$
\begin{gathered}
1-\left\{\frac{1}{4 b^{2}}\left[\left(x_{i}-x_{k}\right)^{2}-\left(y_{i}-y_{k}\right)^{2}\right]+\frac{1}{4 a^{2}}\left[\left(x_{i}+x_{k}\right)^{2}-\left(y_{i}+y_{k}\right)^{2}\right]\right\} \\
=1-\left\{-\frac{4 y_{i}^{2}}{4 b^{2}}+\frac{4 x_{i}^{2}}{4 a^{2}}\right\}<0 .
\end{gathered}
$$

This implies that

$$
\frac{x_{i}^{2}}{a^{2}}-\frac{y_{i}^{2}}{b^{2}}>1,
$$

where $x_{i}<0$ and $x_{k}<0$ since $\mathrm{A}$ is stable. If $\mathrm{A}$ has a real root, then we let $\lambda_{i}=\lambda_{k}$ so that $x_{i}=x_{k}$ and $y_{i}=y_{k}=0$. It follows that $x_{i}^{2}>a^{2}$ which further implies that $x_{i}<-a$ since $\mathrm{A}$ is stable. Thus, we have $\sigma(A) \subset \mathcal{H}_{\mathcal{L}}(a, b)$.

In statement (ii), we note that eigenvalues of $\mathcal{A}$ can be expressed as $-1+m_{i k}$, where

$$
m_{i k} \triangleq \frac{4 a^{2} b^{2}\left(1+\frac{1}{b^{2}} \lambda_{i} \dot{\lambda_{k}}\right)}{\left(a^{2}+b^{2}\right)\left(\lambda_{i}+\bar{\lambda}_{k}\right)^{2}} .
$$

This result can be seen by transforming $A$ into Jordan form. Let $M \triangleq\left[m_{i k}\right] \in \mathcal{R}^{n \times \pi}$. By using the Hadamard product, it is readily seen that

$$
\begin{gathered}
M=\frac{4 a^{2} b^{2}}{a^{2}+b^{2}}\left[\frac{-1}{\lambda_{i}+\bar{\lambda}_{k}}\right]_{i, k=1, \ldots, n} \circ\left[\frac{-1}{\lambda_{i}+\bar{\lambda}_{k}}\right]_{i, k=1, \ldots, n} \\
\circ\left[1+\frac{1}{b^{2}} \lambda_{i}+\lambda_{k}\right]_{i, k=1, \ldots, n} .
\end{gathered}
$$

Now, using Lemma 2.2 and the fact that the Hadamard product of non-negative definite matrices is a non-negative definite matrix (see Theorem 7.5.3 in Ref. 13), we conclude that $M$ is non-negative definite. From the properties of non-negative definite matrices, it is well known that

$$
m_{i i} m_{k k}-\left|m_{i k}\right|^{2} \geq 0
$$

Now, to prove the sufficiency of $(i i)$, note that since $\sigma(A) \subset$ $\mathcal{H}_{\mathcal{L}}(a, b)$, it follows that

$$
\frac{x_{i}^{2}}{a^{2}}-\frac{y_{i}^{2}}{b^{2}}>1
$$

where $\lambda_{i} \triangleq x_{i}+\mathrm{j} y_{i}$. Thus,

$$
\begin{gathered}
m_{i i}=\frac{4 a^{2} b^{2}\left(1+\frac{1}{b^{2}} \lambda_{i} \bar{\lambda}_{i}\right)}{\left(a^{2}+b^{2}\right)\left(\lambda_{i}+\lambda_{i}\right)^{2}}=\frac{4 a^{2} b^{2}\left(1+\frac{1}{b^{2}}\left(x_{i}^{2}+y_{i}^{2}\right)\right)}{4\left(a^{2}+b^{2}\right) x^{2}} \\
<\frac{a^{2} b^{2}+a^{2}\left(x_{i}^{2}+\frac{b^{2} x_{i}^{2}}{a^{2}}-b^{2}\right)}{\left(a^{2}+b^{2}\right) x_{i}^{2}}=1 .
\end{gathered}
$$

Hence, $\left|m_{i k}\right|^{2} \leq m_{i i} m_{k k}<1$ since $m_{i i}<1$ and $m_{k k}<1$. This implies that

$$
\operatorname{Re}\left\{-1+m_{i k}\right\} \leq-1+\left|m_{i k}\right|<0 \text {. }
$$

Thus, $A$ and $\bar{A}$ are stable. To prove necessary of (ii), we can simply reverse the arguments. This completes the proof of statement (ii).

Lemma 2.3 Let $\sigma(A) \subset \mathcal{H}_{\mathcal{L}}(a, b)$ and $V, V_{h} \in \mathcal{R}^{{ }^{n \times 7}}$ be positive definite matrices. Let $\delta$ and $\gamma$ be given by (1). Then there exist unique $n \times n$ positive definite matrices $Q$, $Q_{h}$ satisfying

$$
\begin{gathered}
0=Q_{h}+\delta\left(A^{2} Q_{h}+Q_{h} A^{2 \mathrm{~T}}\right)+\gamma A Q_{h} A^{\mathrm{T}}+V_{h}, \\
0=A Q+Q A^{\mathrm{T}}+V .
\end{gathered}
$$

Proof. The existence of a positive definite solution to (9) is a well known result and hence the proof is omitted. We now turn to the proof of (8). Here, we will first give a proof for the case in which $a \geq b$. From Proposition 2.2, it follows that $\mathcal{A}$ is stable. By using the 'vec' operator defined in Ref. 12, we obtain

$$
Q_{h}=-\operatorname{vec}^{-1} \mathcal{A}^{-1} \text { vec } V_{h},
$$

which is the unique solution to (8). Furthermore, since $\mathcal{A}$ is stable, the following integral exists

$$
\begin{gathered}
Q_{h}=\int_{0}^{\infty} \operatorname{vec}^{-1} e^{\mathcal{A} t} \operatorname{vec} V_{h} d t \\
=\int_{0}^{\infty} \operatorname{vec}^{-1} e^{\left[I+\delta\left(A^{2} \oplus A^{2}\right)\right] t} e^{\gamma(A \otimes A) t} \operatorname{vec} V_{h} d t \\
=\int_{0}^{\infty} \operatorname{vec}^{-1} e^{\left[I+\delta\left(A^{2} \oplus A^{2}\right)\right] t}\left\{\sum_{k=0}^{\infty} \frac{[\gamma(A \otimes A) t]^{k}}{k !} \operatorname{vec} V_{h}\right\} d t \\
=\int_{0}^{\infty} \operatorname{vec}^{-1} e^{\left[I+\delta\left(A^{2} \oplus A^{2}\right)\right] t} \operatorname{vec}\left\{\sum_{k=0}^{\infty} \frac{(\gamma t)^{k} A^{k} V_{h} A^{k \mathrm{~T}}}{k !}\right\} d t \\
=\int_{0}^{\infty} e^{\left[\frac{1}{2} I+\delta A^{2}\right] t}\left\{\sum_{k=0}^{\infty} \frac{(\gamma t)^{k} A^{k} V_{h} A^{k \mathrm{~T}}}{k !}\right\} e^{\left[\frac{1}{2} I+\delta A^{2}\right]^{\mathrm{T}} t} d t>0,
\end{gathered}
$$

where the last inequality follows from the fact that in this case, that is, $b \leq a$, it follows that $\gamma \geq 0$. Thus (8) has a unique positive definite solution.

For the case in which $a<b$, it follows that $\gamma<0$ and the above proof must be modified. Now we note that ( 8 ) can be written as

$$
\delta(A \oplus A)^{2} \mathcal{A}=-\mathcal{A}
$$

Since $A$ is stable, $A \oplus A$ is also stable and thus (8) ran be written as

$$
-\frac{1}{\delta}(A \oplus A)^{-2} \mathcal{A} \text { vec } Q_{h}=\frac{1}{\delta}(A \oplus A)^{-2} \text { vec } V_{h},
$$

or equivalently

$$
\tilde{A} \operatorname{vec} Q_{h}=\frac{1}{\delta}(A \oplus A)^{-2} \operatorname{vec} V_{h} \text {. }
$$

It can now be seen that there exists an unique $Q_{h}$ which 
satisfies (8) given by

$$
Q_{h}=\frac{1}{\delta} \operatorname{vec}^{-1} \tilde{\mathcal{A}}^{-1}(A \oplus A)^{-2} \operatorname{vec} V_{h} .
$$

Since $\tilde{\mathcal{A}}$ is stable, the integral

$$
\operatorname{vec} Q_{h}=-\frac{1}{\delta} \int_{0}^{\infty} e^{\overline{\mathcal{A}}_{t}}\left[(A \oplus A)^{-2} \operatorname{vec} V_{h}\right] d t
$$

exists. Now, defining $Y_{1}$ by

$$
Y_{1} \triangleq \operatorname{vec}^{-1}\left[(A \oplus A)^{-2} \text { vec } V_{h}\right],
$$

it then follows that $Y_{1}$ satisfies

$$
A^{2} Y_{1}+2 A Y_{1} A^{\mathrm{T}}+Y_{1} A^{2 \mathrm{~T}}=V_{h}
$$

This matrix equation is in fact a combined form of the following two Lyapunov equations

$$
\begin{aligned}
& 0=A Y_{1}+Y_{1} A^{\mathrm{T}}+Y_{0}, \\
& 0=A Y_{0}+Y_{0} A^{\mathrm{T}}+Y_{h} .
\end{aligned}
$$

Hence $Y_{1}>0$ since $V_{h}>0, Y_{0}>0$ and $A$ is stable. Thus, we now have

$$
\begin{gathered}
\operatorname{vec} Q_{h}=-\frac{1}{\delta} \int_{0}^{\infty} e^{\left\{-I-\frac{1}{\delta}(A \oplus A)^{-2}[I+(\gamma-2 \delta) A \otimes A]\right\} t} \operatorname{vec} Y_{1} d t \\
=-\frac{1}{\delta} \int_{0}^{\infty} e^{-t} e^{\Phi t} \operatorname{vec} Y_{1} d t \\
=-\frac{1}{\delta} \int_{0}^{\infty} e^{-t}\left[\sum_{k=0}^{\infty} \frac{(\Phi t)^{k}}{k !}\right] \operatorname{vec} Y_{1} d t
\end{gathered}
$$

where $\Phi$ is defined by

$$
\Phi \triangleq-\frac{1}{\delta}(A \oplus A)^{-2}[I+(\gamma-2 \delta) A \otimes A] .
$$

Now, when $\mathrm{k}=1$, we define $Z_{1}$ such that

$$
\operatorname{vec} Z_{1} \triangleq \Phi \operatorname{vec} Y_{1}
$$

which further implies that $Z_{1}$ satisfies

$$
A^{2} Z_{1}+2 A Z_{1} A^{\mathrm{T}}+Z_{1} A^{2 \mathrm{~T}}=-\frac{1}{\delta}\left[Y_{1}+(\gamma-2 \delta) A Y_{1} A^{\mathrm{T}}\right] .
$$

Note that since $\frac{-1}{\delta}>0$ and $\gamma-2 \delta=\frac{1}{b^{2}}>0$, the right hand side of the matrix equation is non-negative definite. The equation can be written as the pair of equations

$$
\begin{gathered}
0=A Z_{1}+Z_{1} A^{\mathrm{T}}+Z_{0} \\
0=A Z_{0}+Z_{0} A^{\mathrm{T}}-\frac{1}{\delta}\left[Y_{1}+(\gamma-2 \delta) A Y_{1} A^{\mathrm{T}}\right] .
\end{gathered}
$$

Thus, $Z_{1}$ is positive definite since $Y_{1}$ and $Z_{0}$ are positive definite and $A$ is stable. When $k=2$, we simply definite $Z_{2}$ such that

$$
\text { vec } Z_{2} \triangleq \Phi \text { vec } Z_{1}=\Phi^{2} \text { vec } Y_{1} \text {. }
$$

Using the same arguments, it then follows that $Z_{2}$ is positive definite since $Z_{1}$ is positive definite. Thus we conclude that

$$
\operatorname{vec}^{-1}\left(\Phi^{k} \operatorname{vec} Y_{1}\right)>0
$$

for each integer $k$. Thus,

$Q_{h}=-\frac{1}{\delta} \int_{0}^{\infty} e^{-t}\left\{\sum_{k=0}^{\infty} \operatorname{vec}^{-1} \frac{(\Phi t)^{k}}{k !}\right\}\left[(A \oplus A)^{-2} \operatorname{vec} V_{h}\right] d t>0$, since $V_{h}$ is positive definite. This completes the proof.

\section{Controller Synthesis With Static Output Feedback}

We consider the linear time-invariant system

$$
\begin{gathered}
\dot{x}(t)=A x(t)+B u(t)+D_{1} w(t), \\
y(t)=C x(t),
\end{gathered}
$$

where $x(t), u(t), w(t)$ and $y(t)$ are $n, m, d$ and $l$-dimensional vectors and $A, B, C, D_{1}$ are corresponding constant matrices. In this section, we consider static output feedback of the form

$$
u(t)=K y(t)
$$

Then the goal is to select $K$ such that the closed-loop system has the following properties:

(i) the closed-loop poles are constrained to lie in the hyperbolic constraint region $\mathcal{H}_{\mathcal{C}}(a, b)$;

(ii) the performance index

$J \triangleq \lim _{t \rightarrow \infty} \mathcal{E} \frac{1}{t} \int_{0}^{t}\left[x(t)^{\mathrm{T}} R_{1} x(t)+2 x(t)^{\mathrm{T}} R_{12} u(t)+u(t)^{\mathrm{T}} R_{2} u(t)\right] d t$

is minimized.

Now, for the closed-loop system (10)-(12), we have

$$
\dot{x}(t)=A_{s} x(t)+D_{1} w(t) .
$$

Then, to determione a feedback gain $K$ satisfying ( $i$ ) and (ii), we begin by defining an open set of feedback gains

$$
\mathcal{K}_{s} \triangleq\left\{K: \sigma\left(A_{s}\right) \subset \mathcal{H}_{\mathcal{L}}(a, b)\right\}
$$

which places the closed-loop poles in $\mathcal{H}_{\mathcal{L}}(a, b)$. We assume that $\mathcal{K}_{s}$ is not empty. Equation (13) can be written as

$$
J(K)=\lim _{t \rightarrow \infty} \mathcal{E}\left(x^{\mathrm{T}} R_{s} x\right)
$$

Furthermore, by defining the non-negative definite state covariance

$$
Q \triangleq \lim _{t \rightarrow t} \mathcal{E}\left(x x^{\mathrm{T}}\right)
$$

the system (10)-(12) combined with criteria (ii) will be: minimize

$$
J(K)=\operatorname{tr} Q R_{s}
$$

subject to

$$
0=A_{s} Q+Q A_{s}^{\mathrm{T}}+V_{s} .
$$

However, in order to impose criteria (ii), we may overbound the desired performance index as shown in the following lemma so that a minimization procedure can be carried out later.

Lemma 3.1 Let $K \in \mathcal{K}_{s}$ and let $V_{s}, V_{h} \in \mathcal{R}^{n \times n}$ be positive definite matrices. Then there exist $n \times n$ positive definite matrices $Q, Q_{h}$ satisfying

$$
\begin{gathered}
0=Q_{h}+\delta\left(A_{s}^{2} Q_{h}+Q_{h} A_{s}^{2 \mathrm{~T}}\right)+\gamma A_{s} Q_{h} A_{s}^{\mathrm{T}}+V_{h}, \\
0=A_{s} Q+Q A_{s}^{\mathrm{T}}+V_{s} .
\end{gathered}
$$

Furthermore,

$$
J(K)<\mathcal{J}(K)
$$

where

$$
\mathcal{J}(K) \triangleq \operatorname{tr}\left(Q R_{s}+Q_{h}\right)
$$


Now, we may formulate the Auxiliary Minimization Prob. lem: determine $K \in \mathcal{K}_{s}$ that minimizes $\mathcal{J}(\mathrm{K})$ where the positive definite matrices $Q_{h}$ and $Q$ satisfy (19) and (20).

Theorem 3.1 Let $K \in \mathcal{K}_{s}$ minimize $\mathcal{J}(K)$. Then there exist positive definite matrices $Q_{h}, Q, P_{h}, P_{s} \in \mathcal{R}^{n \times n}$ satisfying

$$
\begin{gathered}
0=\hat{A} Q+Q \hat{A}^{\mathrm{T}}+V_{s}, \\
0=Q_{h}+\delta\left(\hat{A}^{2} Q_{h}+Q_{h} \hat{A}^{2 \mathrm{~T}}\right)+\gamma \hat{A} Q_{h} \hat{A}^{\mathrm{T}}+V_{h}, \\
0=\hat{A}^{\mathrm{T}} P+P \hat{A}+\hat{R}_{s}, \\
0=I+\delta\left(\hat{A}^{2 \mathrm{~T}} P_{h}+P_{h} \hat{A}^{2}\right)+\gamma \hat{A}^{\mathrm{T}} Q_{h} \hat{A}+P_{h},
\end{gathered}
$$

where, under the assumption that $\Pi$ defined below is nonsingular,

$$
\begin{gathered}
\hat{A} \triangleq A-B\left(\operatorname{vec}^{-1} \Pi^{-1} \operatorname{vec} \Omega\right) C, \\
\hat{R}_{s} \triangleq R_{1}-R_{12}\left(\operatorname{vec}^{-1} \Pi^{-1} \operatorname{vec} \Omega\right) C \\
-C^{\mathrm{T}}\left(\operatorname{vec}^{-1} \Pi^{-1} \operatorname{vec} \Omega\right)^{\mathrm{T}} R_{12}^{\mathrm{T}} \\
+C^{\mathrm{T}}\left(\operatorname{vec}^{-1} \Pi^{-1} \operatorname{vec} \Omega\right)^{\mathrm{T}} R_{2}\left(\operatorname{vec}^{-1} \Pi^{-1} \operatorname{vec} \Omega\right) C, \\
\Omega \triangleq R_{12}^{\mathrm{T}} Q C^{\mathrm{T}}+\delta\left(B^{\mathrm{T}} A^{\mathrm{T}} P_{h} Q_{h} C^{\mathrm{T}}+B^{\mathrm{T}} P_{h} Q_{h} A^{\mathrm{T}} C^{\mathrm{T}}\right) \\
+\gamma B^{\mathrm{T}} P_{h} A Q_{h} C^{\mathrm{T}}+B^{\mathrm{T}} P Q C^{\mathrm{T}}, \\
\Pi \triangleq C Q C^{\mathrm{T}} \otimes R_{2}+\delta\left[\left(C Q_{h} P_{h} B \otimes B^{\mathrm{T}} C^{\mathrm{T}}\right) U_{m \times l}\right. \\
\left.+\left(C B \otimes B^{\mathrm{T}} P_{h} Q_{h} C^{\mathrm{T}}\right) U_{m \times l}\right]+\gamma\left(C Q_{h} C^{\mathrm{T}} \otimes B^{\mathrm{T}} P_{h} B\right),
\end{gathered}
$$

such that the feedback gain $K$ is given by

$$
K=-\operatorname{vec}^{-1} \Pi^{-1} \operatorname{vec} \Omega .
$$

Proof. We first formulate the Lagrangian

$$
\begin{aligned}
\mathcal{L}\left(K, Q, Q_{h}\right)=\operatorname{tr} & \left\{Q R_{s}+Q_{h}+P_{h}\left[Q_{h}+\delta\left(A_{s}^{2} Q_{h}+Q_{h} A_{s}^{2 \mathrm{~T}}\right)\right.\right. \\
& \left.\quad+\gamma A_{s} Q_{h} A_{s}^{\mathrm{T}}+V_{h}\right] \\
+ & \left.P\left(A_{s} Q+Q A_{s}^{\mathrm{T}}+V_{s}\right)\right\}
\end{aligned}
$$

Then, setting the partials of $\mathcal{L}$ with respect to $Q$ and $Q_{h}$ to zero imply that

$$
\begin{gathered}
0=R_{s}+A_{s}^{\mathrm{T}} P+P A_{s}, \\
0=I+P_{h}+\delta\left(A_{s}^{2 \mathrm{~T}} P_{h}+P_{h} A_{s}^{2}\right)+\gamma A_{s}^{\mathrm{T}} P_{h} A_{s} .
\end{gathered}
$$

Similarly, by setting the partial of $\mathcal{L}$ with respect to $K$ to zero, yields (27). Using (27) to replace $K$ in (19)-(20) and (29)-(30), we then obtain (23)-(26).

\section{Suboptimal Full-Order Compensation With Regulator/Estimator Separation}

In this section, we consider the linear time-invariant system

$$
\begin{gathered}
\dot{x}(t)=A x(t)+B u(t)+D_{1} w(t), \\
y(t)=C x(t)+D_{2} w(t),
\end{gathered}
$$

where $x(t), u(t), w(t)$ and $y(t)$ are $n, m, d$ and $l$-dimensional vectors and $A, B, C, D_{1}$ and $D_{2}$ are corresponding constant matrices. Now, the goal is to choose $A_{c}, B_{c}, C_{c}$ such that the dynamic compensator

$$
\begin{gathered}
\dot{x}_{c}(t)=A_{c} x_{c}+B_{c} y(t), \\
u(t)=C_{c} x_{c},
\end{gathered}
$$

satisfies properties (i) and (ii) in Section III.
The closed-loop system (31)-(34) and performance criterion (13) can be restated as: minimize

$$
J\left(A_{\mathfrak{c}}, B_{\mathrm{c}}, C_{c}\right)=\operatorname{tr} Q R_{d},
$$

subject to

$$
0=A_{d} Q+Q A_{d}^{\mathrm{T}}+V_{d} .
$$

As in Section III, the set of dynamic compensators that places the closed-loop poles in $\mathcal{H}_{\mathcal{L}}(a, b)$ is defined by

$$
\mathcal{K}_{d} \triangleq\left\{\left(A_{c}, B_{c}, C_{c}\right): \sigma\left(A_{d}\right) \subset \mathcal{H}_{\mathcal{L}}(a, b)\right\} .
$$

The following result is analogous to Lemma 3.1.

Lemma 4.1 Let the triple $\left(A_{c}, B_{c}, C_{c}\right) \in \mathcal{K}_{d}$ and let $V_{d}, V_{h} \in \mathcal{R}^{n \times n}$ be positive definite matrices. Then there exist positive definite matrices $Q, Q_{h} \in \mathcal{R}^{n \times n}$ satisfying

$$
\begin{gathered}
0=Q_{h}+\delta\left(A_{d}^{2} Q_{h}+Q_{h} A_{d}^{2 \mathrm{~T}}\right)+\gamma A_{d} Q_{h} A_{d}^{\mathrm{T}}+V_{h}, \\
0=A_{d} Q+Q A_{d}^{\mathrm{T}}+V_{d} .
\end{gathered}
$$

Furthermore,

$$
J\left(A_{c}, B_{c}, C_{c}\right)<\mathcal{J}\left(A_{c}, B_{c}, C_{c}\right),
$$

where

$$
\mathcal{J}\left(A_{c}, B_{c}, C_{c}\right) \triangleq \operatorname{tr}\left(Q R_{d}+Q_{h}\right)
$$

Here we enforce regulator/estimator separation for determining $\left(A_{c}, B_{c}, C_{c}\right)$. Thus, the dynamic compensator is assumed to be of the form

$$
\begin{gathered}
\dot{x}_{c}=A x_{c}+B u+B_{c}\left(y-C x_{c}\right), \\
u=C_{c} x_{c} .
\end{gathered}
$$

such that $A_{c} \triangleq A+B C_{c}-B_{c} C$. To exploit this, it is useful to design the estimator by defining the tracking error $e \triangleq x-x_{c}$ such that

$$
\left[\begin{array}{c}
\dot{x} \\
\dot{e}
\end{array}\right]=\left[\begin{array}{cc}
A+B C_{c} & -B C_{c} \\
0 & A+B_{c} C
\end{array}\right]\left[\begin{array}{l}
x \\
e
\end{array}\right]+\left[\begin{array}{c}
D_{1} \\
D_{1}-B_{c} D_{2}
\end{array}\right] w .
$$

Then the goal is to separately place the eigenvalues of the error dynamics and regulator in the hyperbolic constraint region $\mathcal{H}_{\mathcal{L}}(a, b)$. From (43), it is noticed that there are in fact two separate problems for determining $B_{c}$ and $C_{c}$. The subproblem for the estimator can be formulated such that the weighted estimator cost is given by

$$
J_{e}\left(B_{c}\right)=\lim _{t \rightarrow \infty} \mathcal{E}\left(e^{\mathrm{T}} W e\right)
$$

where $W$ is a given $n \times n$ positive definite matrix. However, (44) can be rewritten as

$$
J_{e}\left(B_{c}\right)=\mathrm{tr} Q_{e} W .
$$

Note that $Q_{e}$ satisfies the Lyapunov equation

$$
0=A_{e} Q_{e}+Q_{e} A_{e}^{\mathrm{T}}+V_{e} .
$$

For the regulator, we consider

$$
\begin{gathered}
\dot{x}=A x+B u+D_{1} w, \\
u=C_{c} x .
\end{gathered}
$$

which implies that

$$
\dot{x}=A_{\tau} x+D_{1} w .
$$


The corresponding cost is

$$
\begin{gathered}
J_{r}\left(C_{c}\right)=\lim _{t \rightarrow \infty} \mathcal{E}\left(x^{\mathrm{T}} R_{1} x+2 x^{\mathrm{T}} R_{12} u+u^{\mathrm{T}} R_{2} u\right) \\
=\operatorname{tr} Q_{r} R_{r},
\end{gathered}
$$

where $Q_{r}$ satisfies

$$
0=A_{r} Q_{r}+Q_{r} A_{r}^{\mathrm{T}}+V_{1}
$$

Now, let $\tilde{\mathcal{K}}_{s}$ be defined as follows to characterize a dual set of gains for closed-loop pole assignment.

$$
\overline{\mathcal{K}}_{s} \triangleq\left\{K: \sigma\left(A+B_{\mathrm{c}} C\right) \subset \mathcal{H}_{\mathcal{L}}(a, b) \subset C^{-}\right\}
$$

Remark 4.1 Note that the results derived for full state feedback in Section III can be utilized here to determine the regulator gain $C_{c}$. Nevertheless, the same procedure given in Section III can also be followed to determine the estimator gain $B_{c}$ since it is essentially dual to the problem formulated in Section III

Remark 4.2 To place the eigenvalues of error dynamics and regulator in $\mathcal{H}_{\mathcal{L}}(a, b)$, it is also required that $A_{e}$ and $A_{\tau}$ be stable which directly follow the results of Proposition 2.2 .

Lemma 4.2 Let $B_{c} \in \tilde{\mathcal{K}}_{s}, C_{c} \in \mathcal{K}_{s}$ and let $V_{e}, V_{h e}, V_{1}$, $V_{h r} \in \mathcal{R}^{n \times n}$ be positive definite matrices. Then, there exist positive definite matrices $Q_{h e}, Q_{e} \in \mathcal{R}^{n \times n}$ satisfying

$$
\begin{gathered}
0=Q_{h e}+\delta\left(A_{e}^{2} Q_{h e}+Q_{h e} A_{e}^{2 \mathrm{~T}}\right)+\gamma A_{e} Q_{h e} A_{e}^{\mathrm{T}}+V_{h e}, \\
0=A_{e} Q_{e}+Q_{e} A_{e}^{\mathrm{T}}+V_{e} .
\end{gathered}
$$

such that (45) satisfies the bound

$$
J_{e}\left(B_{c}\right)<\mathcal{J}_{e}\left(B_{c}\right)=\operatorname{tr}\left(Q_{e} W+Q_{h e}\right) .
$$

Furthermore, there exist positive definite matrices $Q_{h r}, Q_{r} \in$ $\mathcal{R}^{n \times n}$ satisfying

$$
\begin{gathered}
0=Q_{h r}+\delta\left(A_{r}^{2} Q_{h r}+Q_{h r} A_{r}^{2 \mathrm{~T}}\right)+\gamma A_{r} Q_{h r} A_{r}^{\mathrm{T}}+V_{h r}, \\
0=A_{r} Q_{r}+Q_{r} A_{r}^{\mathrm{T}}+V_{1} .
\end{gathered}
$$

such that (45) satisfies the bound

$$
J_{r}\left(C_{c}\right)<\mathcal{J}_{r}\left(C_{c}\right)=\operatorname{tr}\left(Q_{r} R_{r}+Q_{h r}\right) .
$$

Proof. Since $A_{e}$ and $A_{\tau}$ are stable, it is well known that $Q_{e}>0, Q_{\tau}>0$ given that $V_{1}>0, V_{e}>0$. To prove (52) and (55), we simply follow the fact that Proposition 2.2 can also be implemented here. Thus, similar to the proof of Lemma 2.3, (52) and (55) follows with no difficulties. Inequalities (54) and (57) hold since $Q_{h e}>0$ and $Q_{h r}>0$.

Theorem 4.1 Let $B_{c} \in \tilde{\mathcal{K}}_{s}, C_{c} \in \mathcal{K}_{s}$ where $\mathcal{J}_{c}\left(B_{c}\right)$ and $\mathcal{J}_{r}\left(C_{c}\right)$ are minimized and let $V_{e}, V_{h e}, V_{1}, V_{h r} \in \mathcal{R}^{n \times n}$ be positive definite matrices. Then, there exist positive definite matrices $P_{h e}, P_{e}, Q_{e}, Q_{h e} \in \mathcal{R}^{n \times n}$ satisfying

$$
\begin{gathered}
0=\hat{A}_{e} Q_{e}+Q_{e} \hat{A}_{e}^{\mathrm{T}}+V_{e} . \\
0=Q_{h e}+\delta\left(\hat{A}_{e}^{2} Q_{h e}+Q_{h e} \hat{A}_{e}^{2 \mathrm{~T}}\right)+\gamma \hat{A}_{e} Q_{h e} \hat{A}_{e}^{\mathrm{T}}+V_{h e}, \\
0=\hat{A}_{e}^{\mathrm{T}} P_{e}+P_{e} \hat{A}_{e}+W . \\
0=I+P_{h e}+\delta\left(\hat{A}_{e}^{2 \mathrm{~T}} P_{h e}+P_{h e} \hat{A}_{e}^{2}\right)+\gamma \hat{A}_{e}^{\mathrm{T}} P_{h e} \hat{A}_{e},
\end{gathered}
$$

and positive definite matrices $P_{h r}, P_{r}, Q_{r}, Q_{h r} \in \mathcal{R}^{n \times n}$ satisfying

$$
\begin{gathered}
0=\hat{A}_{r} Q_{r}+Q_{r} \hat{A}_{r}^{\mathrm{T}}+V_{1} . \\
0=Q_{h r}+\delta\left(\hat{A}_{r}^{2} Q_{h r}+Q_{h r} \hat{A}_{r}^{2 \mathrm{~T}}\right)+\gamma \hat{A}_{r} Q_{h r} \hat{A}_{r}^{\mathrm{T}}+V_{h r}, \\
0=\hat{A}_{r}^{\mathrm{T}} P_{r}+P_{r} \hat{A}_{r}+\hat{R}_{r} . \\
0=I+P_{h r}+\delta\left(\hat{A}_{r}^{2 \mathrm{~T}} P_{h r}+P_{h r} \hat{A}_{r}^{2}\right)+\gamma \hat{A}_{r}^{\mathrm{T}} P_{h r} \hat{A}_{r},
\end{gathered}
$$

where, under the assumption that $\Pi_{e}$ and $\mathrm{II}_{r}$ defined below are non-singular matrices,

$$
\begin{aligned}
& \hat{A}_{e} \triangleq A-\left(\operatorname{vec}^{-1} \Pi_{e}^{-1} \operatorname{vec} \Omega_{\epsilon}\right) C, \\
& \hat{A}_{r} \triangleq A-B\left(\operatorname{vec}^{-1} \Pi_{r}^{-1} \operatorname{vec} \Omega_{r}\right),
\end{aligned}
$$

$$
\hat{R}_{s} \triangleq R_{1}-R_{12} \operatorname{vec}^{-1} \Pi_{\tau}^{-1} \operatorname{vec} \Omega_{r}-\left(\operatorname{vec}^{-1} \Pi_{r}^{-1} \operatorname{vec} \Omega_{r}\right)^{\mathrm{T}} R_{12}^{\mathrm{T}}
$$$$
+\left(\operatorname{vec}^{-1} \Pi_{r}^{-1} \operatorname{vec} \Omega_{r}\right)^{\mathrm{T}} R_{2}\left(\operatorname{vec}^{-1} \Pi_{r}^{-1} \operatorname{vec} \Omega_{r}\right) \text {. }
$$$$
\Omega_{e} \triangleq \delta\left(A^{\mathrm{T}} P_{h e} Q_{h e} C^{\mathrm{T}}+P_{h e} Q_{h e} A^{\mathrm{T}} C^{\mathrm{T}}\right)
$$$$
+\gamma P_{h e} A Q_{h e} C^{\mathrm{T}}+P_{e} Q_{e} C^{\mathrm{T}}-P_{e} D_{1} D_{2}^{\mathrm{T}} \text {, }
$$

$$
\Pi_{e} \triangleq \delta\left(C \otimes\left(P_{h e} Q_{h e} C^{\mathrm{T}}\right) U_{n \times !}+\left[\left(C Q_{h e} P_{h e}\right) \otimes C^{\mathrm{T}}\right] C_{n \times l}\right)
$$$$
+\gamma\left(C Q_{h e} C^{\mathrm{T}}\right) \otimes P_{h e}+\left(D_{2} D_{2}^{\mathrm{T}}\right) \otimes P_{e},
$$$$
\Omega_{r} \triangleq R_{12}^{\mathrm{T}} Q_{r}+\delta\left(B^{\mathrm{T}} A^{\mathrm{T}} P_{h r} Q_{h r}+B^{\mathrm{T}} P_{h r} Q_{h r} A^{\mathrm{T}}\right)
$$$$
+\gamma B^{\mathrm{T}} P_{h r} A Q_{h_{h r}}+B^{\mathrm{T}} P_{r} Q_{r},
$$$$
\Pi_{r} \triangleq Q_{r} \otimes R_{2}+\delta\left[\left(B \otimes B^{\mathrm{T}} P_{h r} Q_{h r}\right) U_{m \times n}\right.
$$$$
\left.+\left(Q_{h r} P_{h r} B \otimes B^{\mathrm{T}}\right) U_{m \times n}\right]+\gamma Q_{h r} \diamond B^{\mathrm{T}} P_{h r} B,
$$

such that the compensator is given by

$$
\begin{gathered}
A_{c}=A-B_{c} C+B C_{c}, \\
B_{c}=-\operatorname{vec}^{-1} \Pi_{e}^{-1} \operatorname{vec} \Omega_{e}, \\
C_{c}=-\operatorname{vec}^{-1} \Pi_{r}^{-1} \operatorname{vec} \Omega_{r} .
\end{gathered}
$$

Proof. Note that we separately design $B_{c}$ and $C_{c}$ where $B_{c}$ and $C_{c}$ are simply regarded as gains. Thus the proof is similar to the proof given in Theorem 3.1 and hence is omitted.

Remark 4.3 The closed-loop cost satisfies

$$
J\left(A_{c}, B_{c}, C_{c}\right) \leq \mathcal{J}\left(A_{c}, B_{c}, C_{c}\right),
$$

where

$$
\begin{array}{r}
\mathcal{J}\left(A_{c}, B_{c}, C_{c}\right)=\operatorname{tr} Q\left[\begin{array}{c}
R_{1} \\
-\left(R_{12} \operatorname{vec}^{-1} \Pi_{r}^{-1} \operatorname{vec} \Omega_{r}\right)^{\mathrm{T}} \\
-R_{12} \operatorname{vec}^{-1} \mathrm{II}_{r}^{-1} \operatorname{vec}^{-1} \Omega_{r} \\
\left(\operatorname{vec}^{-1} \Pi_{r}^{-1} \operatorname{vec} \Omega_{r}\right)^{\mathrm{T}} R_{2}\left(\operatorname{vec}^{-1} \Pi_{r}^{-1} \operatorname{vec} \Omega_{r}\right)
\end{array}\right],
\end{array}
$$

and $Q$ is given by (35).

\section{Suboptimal Fixed-Order Compensator With an Optimal Projection Condition}

In this section we do consider separation. We will minimize the performance bound defined in (40) with the constraint equations (37), (38) and follow an approach as of Section III. The problem addressed in this section is more general than that of Section IV in that we now consider both full and reduced order compensators. We will first consider the reduced order case in which the compensator is constructed with dimension less than that of the state vector. The full order case then follows as a special case. 
In optimizing with respect to $A_{c}$, it turns out that the equation which determines $A_{c}$, which is a function of $B_{c}$ and $C_{c}$, also includes $A_{c}^{\mathrm{T}}$. Thus it is difficult to express $A_{c}$ as a function $B_{c}$ and $C_{c}$ unless we impose some conditions to simplify the problem. Here, we impose an optimal projection condition such that the terms involving $A_{c}^{\mathrm{T}}$ vanish in the equation. The projection condition is completely analogous to that given in Ref. 11. Thus, the following results will be suboptimal.

Lemma 5.1 Let $\hat{Q}$ and $\hat{P}$ be $n \times n$ non-negative definite matrices and suppose that rank $\hat{Q} \hat{P}=n_{c}$. Then there exist unique $n_{c} \times n$ matrices $G, \Gamma$ and an $n_{c} \times n_{c}$ matrix $M$, except for a change of basis in $\mathcal{R}^{n_{c}}$, such that

$$
\begin{gathered}
\hat{Q} \hat{P}=G^{\mathrm{T}} M \Gamma, \\
\Gamma G^{\mathrm{T}}=I_{n_{c}} .
\end{gathered}
$$

Proof. See Ref. 11 .

By the application of Lemma 5.1, we may impose the optimal projection by defining the following quantities so that the derivation for obtaining $\left(A_{c}, B_{c}, C_{c}\right)$ will be greatly simplified. Thus, we define $\left(n+n_{c}\right) \times\left(n+n_{c}\right)$ matrices $P_{h}$ and $Q_{h}$ which are partitioned into $n \times n, n \times n_{c}, n_{c} \times n_{c}$ sublolocks as

$$
P_{h} \triangleq\left[\begin{array}{cc}
P_{h 1} & P_{h 12} \\
P_{h 12}^{\mathrm{T}} & P_{h 2}
\end{array}\right], \quad Q_{h} \triangleq\left[\begin{array}{cc}
Q_{h 1} & Q_{h 12} \\
Q_{h 12}^{\mathrm{T}} & Q_{h 2}
\end{array}\right],
$$

where it is assumed that $P_{h 2}>0, Q_{h 2}>0$. We also define

$$
\begin{gathered}
\Gamma_{h} \triangleq-P_{h 2}^{-1} P_{h 12}^{\mathrm{T}}, \quad G_{h}^{\mathrm{T}} \triangleq Q_{h 12} Q_{h 2}^{-1}, \\
\Gamma \triangleq-P_{2}^{-1} P_{12}^{\mathrm{T}} \quad G^{\mathrm{T}} \triangleq Q_{12} Q_{2}^{-1},
\end{gathered}
$$

and non-negative definite matrices

$$
\begin{aligned}
& Q_{h} \triangleq Q_{h 1}-Q_{h 12} Q_{h 2}^{-1} Q_{h 12}^{\mathrm{T}}, \quad P_{h} \triangleq P_{h 1}-P_{h 12} P_{h 2}^{-1} P_{h 12}^{\Gamma}, \\
& \hat{Q}_{h} \triangleq Q_{h 12} Q_{h 2}^{-1} Q_{h 12}^{\mathrm{T}}, \quad \hat{P}_{h} \triangleq P_{h 12} P_{h 2}^{-1} P_{h 12}^{\mathrm{T}} \\
& Q \triangleq Q_{1}-Q_{12} Q_{2}^{-1} Q_{12}^{\mathrm{T}}, \quad P \triangleq P_{1}-P_{12} P_{2}^{-1} P_{12}^{\mathrm{T}}, \\
& \hat{Q} \triangleq Q_{12} Q_{2}^{-1} Q_{12}^{\mathrm{T}}, \quad \hat{P} \triangleq P_{12} P_{2}^{-1} P_{12}^{\mathrm{T}} \text {. }
\end{aligned}
$$

Theorem 5.1 Let $\left(A_{c}, B_{c}, C_{c}\right) \in \mathcal{K}_{d}$ minimize $\mathcal{T}\left(A_{c}\right.$, $\left.B_{c}, C_{c}\right)$ and let $V_{d}, V_{h} \in \mathcal{R}^{n \times n}$ be positive definite matrices. Then there exist positive definite matrices $P_{h}, Q_{h}, P, Q \in$ $\mathcal{R}^{n \times n}$ satisfying

$$
\begin{gathered}
0=A_{d} Q+Q A_{d}^{\mathrm{T}}+V_{d} . \\
0=Q_{h}+\delta\left(A_{d}^{2} Q_{h}+Q_{h} A_{d}^{2 \mathrm{~T}}\right)+\gamma A_{d} Q_{h} A_{d}^{\mathrm{T}}+V_{h}, \\
0=A_{d}^{\mathrm{T}} P+P A_{d}+R_{d} . \\
0=I+P_{h}+\delta\left(A_{d}^{2 \mathrm{~T}} P_{h}+P_{h} A_{d}^{2}\right)+\gamma A_{d}^{\mathrm{T}} P_{h} A_{d},
\end{gathered}
$$

such that $A_{c}, B_{c}$ and $C_{c}$ are given by

$$
\begin{gathered}
A_{c}=\Gamma_{h} A G_{h}^{\mathrm{T}}+\Gamma_{h} B C_{c}-B_{c} C G_{h}^{\mathrm{T}} \\
-\frac{\delta}{\gamma}\left[P_{h 2}^{-1} C_{c}^{\mathrm{T}} B^{\mathrm{T}} P_{h} G_{h}^{\mathrm{T}}-\Gamma_{h} Q_{h} C^{\mathrm{T}} B_{c}^{\mathrm{T}} Q_{h 2}^{-1}\right], \\
{\left[\begin{array}{l}
B_{c} \\
C_{c}
\end{array}\right]=-\operatorname{vec}\left(\left[\begin{array}{ll}
\Theta_{11} & \Theta_{12} \\
\Theta_{21} & \Theta_{22}
\end{array}\right]^{-1}\left[\begin{array}{c}
\operatorname{vec} \Omega_{1} \\
\operatorname{vec} \Omega_{2}
\end{array}\right]\right),}
\end{gathered}
$$

under the assumption that $\left[\begin{array}{cc}\Theta_{11} & \Theta_{12} \\ \Theta_{21} & \Theta_{22}\end{array}\right]$ is non-singular, where

$$
\begin{gathered}
\Omega \triangleq \triangleq \\
-P_{2} \Gamma Q C^{\mathrm{T}}-P_{2} \Gamma V_{12}-\delta\left(P_{h 2} \Gamma_{h} Q_{h} A^{\mathrm{T}} C^{\mathrm{T}}\right. \\
\left.+G_{h} A^{\mathrm{T}} \hat{P}_{h} Q_{h} C^{\mathrm{T}}\right)-\gamma P_{h 2} \mathrm{C}_{h} A Q_{h} C^{\mathrm{T}}, \\
\Omega 2 \triangleq R_{12}^{\mathrm{T}} G^{\mathrm{T}} Q_{2}+B^{\mathrm{T}} P G^{\mathrm{T}} Q_{2}+\delta\left(B^{\mathrm{T}} A^{\mathrm{T}} P_{h} G_{h}^{\mathrm{T}} Q_{h 2}\right. \\
\left.+B^{\mathrm{T}} P_{h} \hat{Q}_{h} A^{\mathrm{T}} \Gamma_{h}^{\mathrm{T}}\right)+\gamma B^{\mathrm{T}} P_{h} A G_{h}^{\mathrm{T}} Q_{h 2}, \\
\Theta 11 \triangleq V_{2} \otimes P_{2}+\delta\left(C Q_{h} \Gamma_{h}^{\mathrm{T}} P_{h 2} \otimes G_{h} C^{\mathrm{T}}\right. \\
\left.+C G_{h}^{\mathrm{T} \otimes} \otimes P_{h 2} \Gamma_{h} Q_{h} C^{\mathrm{T}}\right) U_{n_{h} \times l} \\
\Theta 12 \triangleq \delta\left(C Q_{h} P_{h} B \otimes I\right) U_{m \times n_{c}}+\frac{\delta^{2}}{\gamma} \Gamma C Q_{h} \Gamma_{h}^{\mathrm{T}} \otimes G_{h} P_{h} B, \\
\Theta 21 \triangleq \delta\left(I \otimes B^{\mathrm{T}} P_{h} Q_{h} C^{\mathrm{T}}\right) U_{n_{c} \times l}+\frac{\delta^{2}}{\gamma} \Gamma_{h} Q_{h} C^{\mathrm{T}} \otimes B^{\mathrm{T}} P_{h} G_{h}, \\
\frac{\delta^{2}}{\gamma} C Q_{h} \hat{P}_{h} Q_{h} C^{\mathrm{T}} \otimes Q_{h 2}^{-1}+\gamma C Q_{h} C^{\mathrm{T}} \otimes P_{h 2}, \\
\Theta_{22} \triangleq Q_{2} \otimes R_{2}+\delta\left(\Gamma_{h} B \otimes B^{\mathrm{T}} P_{h} G_{h}^{\mathrm{T}} Q_{h 2}\right. \\
\left.+Q_{h 2} G_{h} P_{h} B \otimes B^{\mathrm{T}} \Gamma_{h}^{\mathrm{T}}\right) U_{m \times n_{c}} \\
-\frac{\delta^{2}}{\delta} P_{h 2}^{-1} \otimes B^{\mathrm{T}} P_{h} \hat{Q}_{h} P_{h} B+\gamma Q_{h 2} \otimes B^{\mathrm{T}} P_{h} B .
\end{gathered}
$$

Proof. The proof is straightforward but complicated in algebra and hence is omitted.

Remark 5.1 To obtain a full order compensator with the imposition of optimal projection condition, we simply let $\Gamma=\Gamma_{h}=G_{h}^{\mathrm{T}}=G^{\mathrm{T}}=1$. By comparing $A_{c}=A+B C_{c}-B_{c} C$ for the case of separation and

$$
A_{c}=A+B C_{c}-B_{c} C-\frac{\delta}{\gamma}\left(P_{h 2}^{-1} C_{c}^{\mathrm{T}} B^{\mathrm{T}} P_{h}-Q_{h} C^{\mathrm{T}} B_{r}^{\mathrm{T}} Q_{h 2}^{-1}\right),
$$

for the case that enforces optimal projection, we observe that the results from both cases are not directly related.

\section{Characterization of the Horizontal Strip Region}

In this section we will briefly discuss regional pole placement within the horizontal strip region. To guarantee stability, we are only interested in the region which is in the open left half plane. The left portion of the horizontal strip region, as shown in Figure 2, can be characterized as

$$
\mathcal{H}_{s}(\omega) \triangleq\left\{\lambda \in \mathcal{C}: \operatorname{Re} \lambda<0,(\operatorname{Im} \lambda)^{2}<\omega^{2}\right\},
$$

where $\omega$ is the upper frequency bound

Lemma 6.1 The set $\mathcal{H}_{s}(\omega)$ is equivalent to

$$
\mathcal{H}_{s}(\omega)=\left\{\lambda \in \mathcal{C}:-1+\delta \operatorname{Re} \lambda^{2}+\gamma|\lambda|^{2}<0\right\},
$$

where

$$
\delta \triangleq-\frac{1}{4 \omega^{2}}, \quad \gamma \triangleq \frac{1}{2 \omega^{2}} .
$$

Proof. The result can be shown using simple algebra. $\square$

By comparing $\mathcal{H}(a, b)$ defined in Lemma 2.1 and $\mathcal{H}_{s}(\omega)$ defined in Lemma 6.1, we immediately notice that the constraint inequalities are similar. The differences only arise at the coefficients of the inequalities. Thus, the major results derived so far for the hyperbolic constraint region can be carried over to be the results for the horizontal strip region with slight modifications at the coefficients. 


\section{Numerical Example}

Consider the dynamical system shown in Figure 3 which includes mass, spring and damper. The system can be converted into a state equation that involves two states, one input and two outputs. Namely, letting $x_{1}$ be the position vector and $x_{2}$ be the velocity vector, it then follows that

$$
\begin{gathered}
{\left[\begin{array}{c}
\dot{x}_{1} \\
\dot{x}_{2}
\end{array}\right]=\left[\begin{array}{cc}
0 & 1 \\
-0.75 & -0.3
\end{array}\right]\left[\begin{array}{l}
x_{1} \\
x_{2}
\end{array}\right]+\left[\begin{array}{c}
0 \\
1.2
\end{array}\right] u+\left[\begin{array}{l}
0 \\
1
\end{array}\right] w,} \\
{\left[\begin{array}{l}
y_{1} \\
y_{2}
\end{array}\right]=\left[\begin{array}{ll}
1 & 0 \\
0 & 1
\end{array}\right]\left[\begin{array}{l}
x_{1} \\
x_{2}
\end{array}\right] .}
\end{gathered}
$$

The goal is to design a feedback controller such that the closed-loop system maintains settling time less than 20 seconds and damping ratio 0.4 . This performance criteria corresponds to $\eta_{\min }=0.2$ and $\zeta_{\min }=0.4$. Thus the desired hyperbolic region is constrained by

$$
\frac{x^{2}}{0.2^{2}}-\frac{y^{2}}{0.4583^{2}}=1,
$$

where $x, y$ are $\operatorname{Re} \lambda$ and $\operatorname{Im} \lambda$, respectively. It is assumed that

$$
R_{1}=\left[\begin{array}{cc}
0.1 & 0 \\
0 & 0.6
\end{array}\right], \quad R_{12}=0, \quad R_{2}=80,
$$

in order to meet the performance requirement. We shall implement Theorem 4.1 to design a feedback controller for the system. Following the numerical procedures discussed in Ref. 1, we then obtain gains $B_{c}$ and $C_{c}$. It is shown in Figure 4 that the open loop system has poles close to imaginary axis and lightly damped whereas the poles from full-state feedback regulator design are at $-0.156 \pm j 0.87$ and the closed-loop poles, under the hyperbolic region constraint, are located at $-0.402 \pm \mathrm{j} 0.763$ (regulator), $-0.462 \pm \mathrm{j} 0.692$ (estimator). In Figure 5, we show the impulse responses from the full-state feedback regulator design. In Figure 6 , it shows the inpulse responses $y_{1}$ and $y_{2}$ where the separation technique is implemented. It is observed that the design that minimizes a performance measure $\mathcal{J}$ with the closed-loop poles constrained to lie in the left hyperbolic region is better.

\section{Conclusion}

In this paper we have shown that it is possible to establish an upper bound for the cost which can be minimized with a pair of matrix root-clustering equations. These equations are then utilized to characterize the poles of the closed-loop system such that they are all constrained to lie in a hyperbolic or horizontal strip region contained in the left half plane. The left hyperbolic region was chosen because of its ability to set desired bounds on the damping ratio and settling time. It is also shown that due to the similarity between root-clustering equations of hyperbolic and horizontal strip regions, the results obtained for the hyperbolic region can be applied to horizontal strip region with minor coefficient changes. Future research will focus on numerical techniques for solving the matrix algebraic equations.

\section{Acknowledgment}

The authors wish to thank Shaul Gutman and Wassim Haddad for helpful discussions and suggestions.

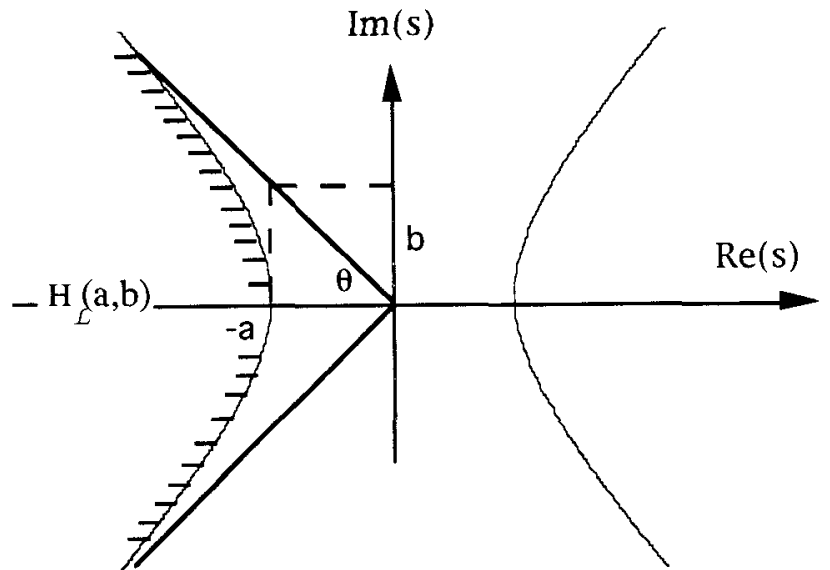

Figure 1 Hyperbolic Region

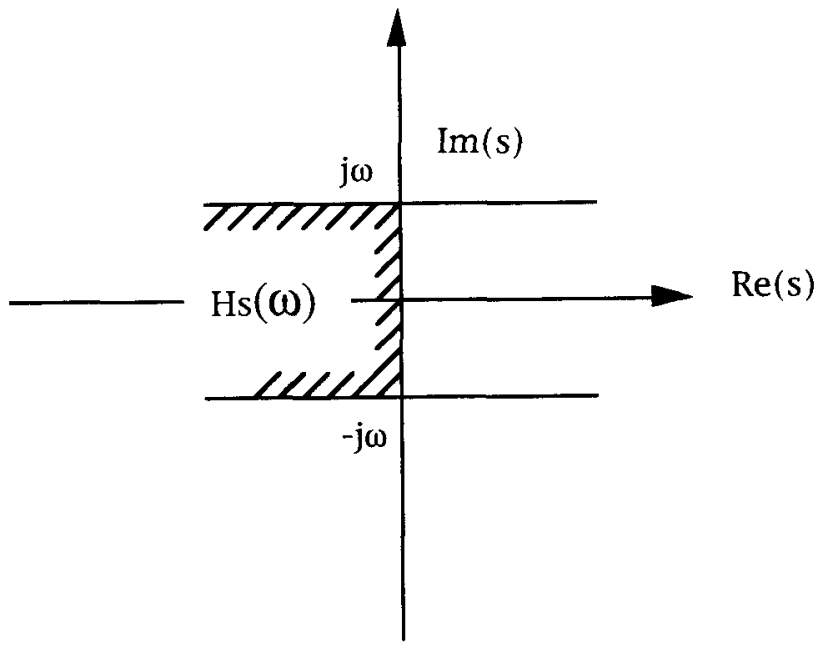

Figure 2 Horizontal Strip Region

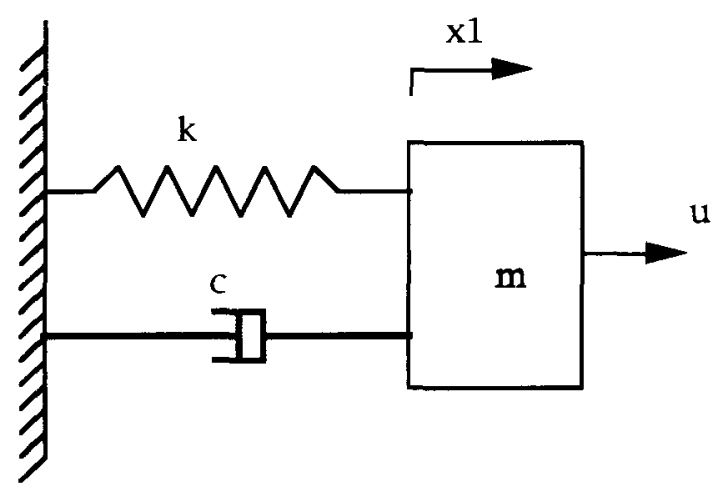

Figure 3 A Simple Dynamical System 


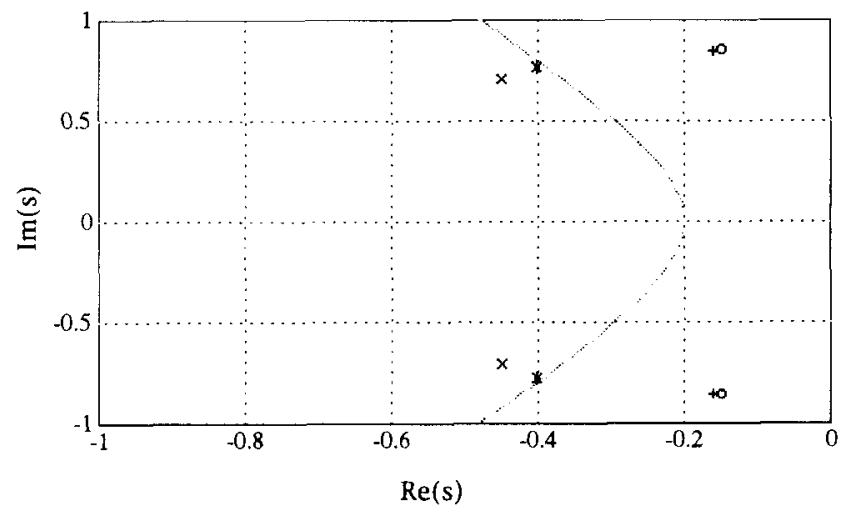

'o'- Open Loop Poles ' + '- Poles of the Unconstrained Regulator '*'- Poles of the Constrained Regulator

' $x$ '- Poles of the Constrained Estimator

Figure 4
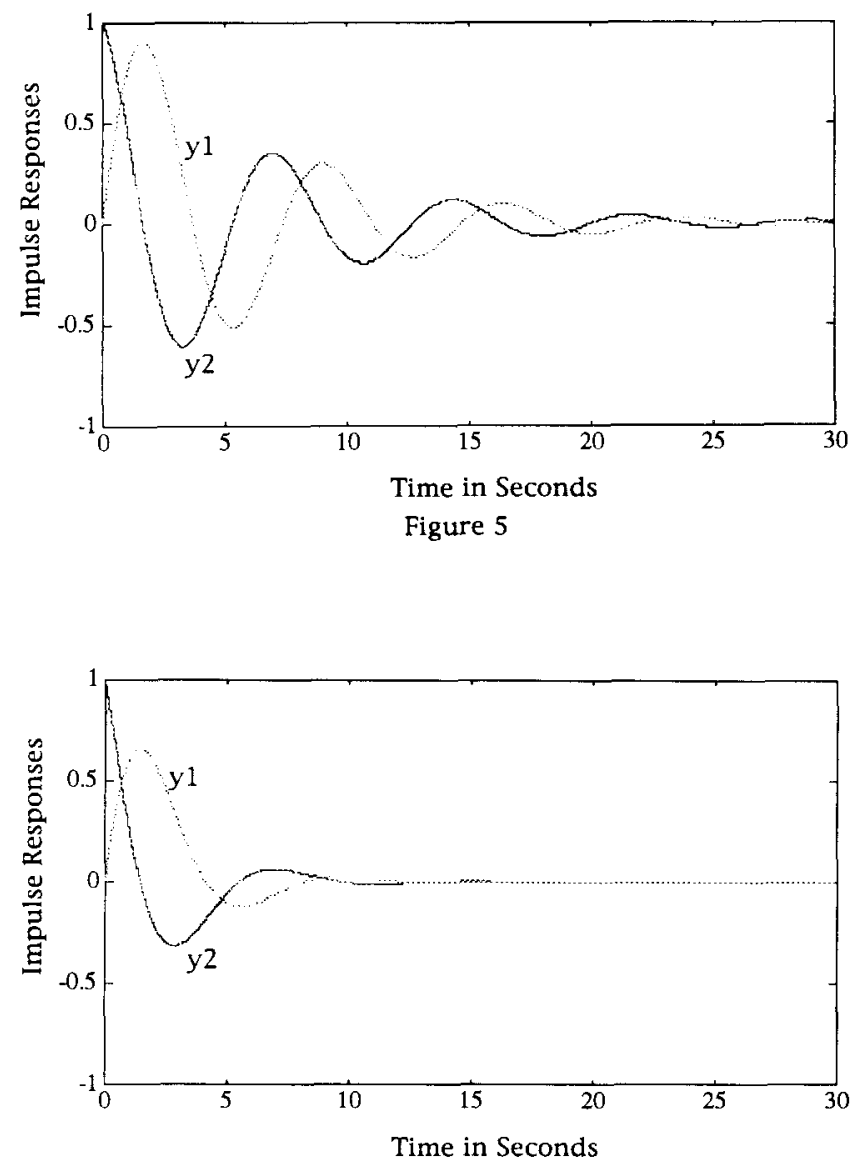

Figure 6

\section{References}

[1] Haddad, W. M., and Bernstein, D. S., "Controller Design with Regional Pole Constraints," IEEE Transactions on Automatic Control, Vol. 37, No. 1, 1992, pp. 54-69.

[2] Gutman, S., and Jury, E. I., "A General Theory for Matrix Root-Clustering in Subregions of the Complex Plane," IEEE Transactions on Automatic Control, Vol. 26, No. 4, 1981, pp. 853-863.

[3] Gutman, S., and Taub, H., "Linear Matrix Equations and Root Clustering," International Journal of Control, Vol. 50, No. 5, 1989, pp. 1635-1643.

[4] Kawasaki, N., and Shimemura, E., "Determining Quadratic Weighting Matrices to Locate Poles in a Specified Region," Automatica, Vol. 19, 1983, pp. 557-560.

[5] Kawasaki, N., and Shimemura, E., "Pole Placement in a Specified Region Based on a Linear Quadratic Regulator," International Journal of Control, Vol. 48, No. 1 , 1988, pp. 225-240.

[6] Gutman, S., Root Clustering in Parameter Space, Lecture Notes in Control and Information Sciences 141, Springer-Verlag, New York, 1990.

[7] Taub, H., and Gutman, S., " A Symmetric Matrix Criterion for Polynomial Root Clustering," IEEE Transactions on Circuits and Systems, Vol. 37, No. 2, 1990, pp. 243-248.

[8] Paldi, E., Paldi, H., and Gutman, S., "The Intersection of Regions and Root Clustering," Linear Algebra and Its Application, Vol. 149, 1991, pp. 151-164.

[9] Zeheb, E., and Hertz, D., "Complete Root Distribution with respect to Parabolas and Some Results with respect to Hyperbolas and Sectors," International Journal of Control, Vol. 36, No. 3, pp. 517-530.

[10] Abdul-Wahab, A., and Zohdy, M. A., "Eigenvalue Clustering in Subregions of the Complex Plane," International Journal of Control, Vol. 48, No. 6, 1988, pp. 25272538.

[11] Hyland, D. C., and Bernstein, D. S., "The Optimal Projection Equations for Fixed-Order Dynamic Compensator," IEEE Transactions on Automatic Control, Vol. 29, No. 11, 1984, pp. 1034-1037.

[12] Brewer, J. W., "Kronecker Products and Matrix Calculus in System Theory," IEEE Transactions on Circuits and Systems, Vol. 25, No. 9, 1978, pp. 772-781.

[13] Horn, R. A., and Johnson, C. A., Matrix Analysis, Cambridge University Press, 1985. 